Research Article

\title{
Metabolic Derangements Contribute to Reduced sRAGE Isoforms in Subjects with Alzheimer's Disease
}

\author{
Kelly N. Z. Fuller, ${ }^{1}$ Edwin R. Miranda, ${ }^{1}$ John P. Thyfault, ${ }^{2}$ Jill K. Morris, ${ }^{3}$ \\ and Jacob M. Haus ${ }^{1}{ }^{1}$ \\ ${ }^{1}$ Department of Kinesiology and Nutrition, University of Illinois at Chicago, Chicago, IL, USA \\ ${ }^{2}$ Department of Molecular \& Integrative Physiology, University of Kansas Medical Center, Kansas City, KS, USA \\ ${ }^{3}$ Department of Neurology, University of Kansas Medical Center, Fairway, KS, USA
}

Correspondence should be addressed to Jacob M. Haus; hausj@uic.edu

Received 15 November 2017; Accepted 9 January 2018; Published 22 February 2018

Academic Editor: Hermann Gram

Copyright (c) 2018 Kelly N. Z. Fuller et al. This is an open access article distributed under the Creative Commons Attribution License, which permits unrestricted use, distribution, and reproduction in any medium, provided the original work is properly cited.

\begin{abstract}
Although there is evidence for metabolic dysfunction and chronic inflammation in Alzheimer's disease (AD), circulating levels of soluble receptor for advanced glycation end products (sRAGE) and the receptor for advanced glycation end products (RAGE) ligand S100B have not been characterized. sRAGE is an important mediator in disease as it can act as a ligand decoy for RAGE and attenuate downstream inflammatory signaling. Cognitively healthy elderly and AD participants with and without type 2 diabetes $(n=135)$ were stratified according to the clinical dementia rating (CDR; $0=$ normal cognition $(\mathrm{NC}) ; \geq 0.5=\mathrm{AD})$. Total serum sRAGE, endogenous secretory RAGE (esRAGE), and S100B were assayed via ELISAs, and cleaved RAGE (cRAGE) and the cRAGE: esRAGE ratio were calculated. cRAGE: esRAGE was lower in AD compared to NC $(p<0.05)$. Metabolic substratifications were used to investigate the factors that influence sRAGE pathology in AD. Stratification by BMI classification, median fat mass, median HOMA-IR, median insulin, and median amylin were all metabolic or anthropometric factors which significantly interacted with sRAGE profiles within AD subjects. There were no significant differences in serum S100B between groups. These characterizations of sRAGE contribute evidence to the link between impaired metabolism and cognitive decline due to $\mathrm{AD}$.
\end{abstract}

\section{Introduction}

Research has identified impaired glucose metabolism and type 2 diabetes (T2DM) as risk factors for cognitive impairment (CI) and Alzheimer's disease (AD) [1-4]. Further, growing evidence of shared biochemical features between $\mathrm{AD}, \mathrm{T} 2 \mathrm{DM}$, and insulin resistance (IR) suggests that $\mathrm{AD}$ may fundamentally be characterized as a metabolic disorder [5-7]. The recent increase in life expectancy has led to a greater population of individuals who are experiencing comorbid T2DM and $\mathrm{AD}$, as prevalence of both T2DM and $\mathrm{AD}$ increases with age $[8,9]$. Therefore, it is important to investigate the cellular and metabolic profiles of these individuals to give insight into the potential mechanisms underlying the onset of $\mathrm{AD}$, which have yet to be fully elucidated. Recent evidence suggests that the receptor for advanced glycation end products (RAGE) may serve as an important mechanistic link [10].

RAGE is a transmembrane receptor of the immunoglobulin superfamily, thought to have evolved from a family of cell adhesion molecules [11]. RAGE has a diverse set of ligands, which include advanced glycation end products (AGEs), beta-amyloid (A $\beta$ ), and members of the S100/calgranulin protein family $[12,13]$. These ligands and the RAGE protein are increased in the postmortem brains of $\mathrm{AD}$ patients, and interactions between RAGE and its ligands are related to the pathophysiology of chronic disease [14, 15]. More specifically, it has been documented that in the $\mathrm{AD}$ brain, there is an overexpression of glial-derived factors including the S100B protein [16]. At high concentrations, S100B accumulates at the surface of RAGE and activates the receptor [17]. Activation of RAGE initiates oxidative 
stress signaling pathways and inflammation through the transcription factor nuclear factor-kappa B (NF- $\kappa \mathrm{B})$ [18]. Chronic inflammation and excessive ROS production are hallmarks of diabetes and metabolic disorders, and increased oxidative stress in the brain is a major inducer of $\mathrm{AD}$ [19-21]. RAGE is further implicated in the pathogenesis of $\mathrm{AD}$ as it plays a critical role in cerebral $\mathrm{A} \beta$ production and accumulation, neuronal degeneration, diminished synaptic transmission, and the formation of fibrous tangles [10]. These biological factors are considered to be the main contributors to the cognitive impairment characteristic of $\mathrm{AD}[22]$.

RAGE exists in two major forms: full-length, membrane bound RAGE and a circulating form termed soluble RAGE (sRAGE). Further, sRAGE is comprised of two distinct isoforms produced by unique mechanisms. Cleaved RAGE (cRAGE) is produced via proteolytic cleavage of the RAGE ectodomain by matrix metalloproteases and endogenous secretory RAGE (esRAGE) results from alternative splicing of RAGE pre-mRNA $[23,24]$. Both isoforms of sRAGE lack the transmembrane and cytosolic domains necessary for signal transduction and act as a ligand decoy, thus attenuating intracellular RAGE signaling and subsequent inflammation [25]. Individuals with obesity, diabetes, or CI alone present with reduced circulating sRAGE profiles compared to lean healthy controls [26-29]. This evidence has led to the emergence of sRAGE as a suggested biological marker of both glucose tolerance status [29] and cognitive decline [30, 31].

Despite the well-established link between RAGE and the pathophysiology of T2DM and AD, the effects of concurrent metabolic and cognitive derangement on sRAGE remain unknown. The purpose of this study was to investigate plasma levels of sRAGE and the RAGE ligand S100B in individuals with metabolic disturbances such as obesity, impaired fasting glucose, elevated amylin, and T2DM comorbid with AD. Our hypothesis was that individuals with concurrent metabolic derangement and $\mathrm{AD}$ would display decreased levels of sRAGE isoforms and elevated levels of S100B. Further, we hypothesized that plasma sRAGE levels would be inversely related to that of S100B.

\section{Methods}

2.1. Participants. All participants $(n=135)$ included in this cross-sectional analysis were part of the University of Kansas Alzheimer's Disease Center Clinical Cohort and provided written informed consent according to the University of Kansas Medical Center's institutional guidelines. Eligible participants were postmenopausal, on stable medicine, and able to provide informed consent. Exclusion criteria included diagnosis of type 1 diabetes, clinically significant depression, or any neurodegenerative disorders other than AD. We a priori included samples from an approximately equal number of T2DM participants in both the ND and AD groups.

2.2. Clinical Characterization and Measurements. Individuals were clinically assessed, and dementia severity was characterized using the Clinical Dementia Rating (CDR). All subjects underwent neuropsychometric testing. Diagnosis was confirmed at a consensus diagnosis conference based on clinical information and neuropsychometric performance. Participants were considered to have normal cognition (NC) if they were CDR 0 with no clinically significant deficits on cognitive testing. All AD subjects were diagnosed as either mild cognitive impairment due to probable AD or dementia due to probable $\mathrm{AD}$ at the time of the blood draw. All AD subjects had a CDR of 0.5 or higher.

For characterization by apolipoprotein $\varepsilon 4$ (APOE4) genotype, DNA was isolated from whole blood and sent to the National Cell Repository for Alzheimer's Disease (NCRAD) for genotyping. Participants were classified as either APOE4 carriers or noncarriers based on the presence of a single APOE4 allele.

American Diabetes Association (ADA) criteria were used to characterize the fasting glucose status (FGS) of participants. Participants were categorized as having normal fasting glucose (NFG, <100 mg/dL), impaired fasting glucose (IFG, $100-125 \mathrm{mg} / \mathrm{dL}$ ), or diabetes (T2DM, >125 mg/ $\mathrm{dL})$. Further, homeostatic model assessment of insulin resistance (HOMA-IR) was computed as a measure of insulin sensitivity [32].

World Health Organization (WHO) criteria were used to characterize the body mass index (BMI) of participants. Participants were classified as lean $\left(18.5-24.9 \mathrm{~kg} / \mathrm{m}^{2}\right)$, overweight $\left(25-29.9 \mathrm{~kg} / \mathrm{m}^{2}\right)$, or obese $\left(>30 \mathrm{~kg} / \mathrm{m}^{2}\right)$. Body composition was assessed via dual-energy X-ray absorptiometry (DEXA, Lunar Prodigy, version 11.2068) for measures of lean body mass and fat mass.

2.3. Serum Analysis. Participants reported to the clinic following an overnight fast, and blood was collected via venipuncture into serum vacutainer tubes containing clot activator. Blood was processed for serum, and glucose was measured using a YSI 2300 Stat Plus Glucose Lactate Analyzer. All remaining serum was stored at $-80^{\circ} \mathrm{C}$ until further analysis. Insulin (Genway), amylin (Millipore), S100B (Millipore), sRAGE (R\&D), and esRAGE (As One International) were quantified using ELISA per the manufacturer's protocol. Serum cRAGE was computed by subtracting esRAGE from the total plasma sRAGE pool as previously described [33], and the cRAGE: esRAGE ratio was subsequently calculated [29].

2.4. Statistics. All data were tested for normality using Shapiro-Wilk's test. Nonnormally distributed data was logtransformed and retested for normality. The Student's $t$-test was used to compare serum sRAGE and S100B profiles between the $\mathrm{AD}$ and $\mathrm{NC}$ groups. An analysis of variance (ANOVA) and Bonferroni post hoc analysis were used to determine significant differences in sRAGE isoforms between cognition groups and across important metabolic factors. An analysis of covariance (ANCOVA) was used following the ANOVA to determine the effects of age and sex, two wellestablished influences in AD pathology. Pearson's correlation coefficient was used to determine bivariate correlation analyses. All data are presented as mean \pm SEM, and differences were determined statistically significant at a $p$ value $<0.05$. 


\section{Results}

3.1. Subject Characteristics. Cognition groups were weighted equally with T2DM participants. There were no significant differences between cognition groups with respect to fasting glucose, fasting insulin, or HOMA-IR (Table 1). There was also no difference in age or lean body mass between groups; however, the $\mathrm{AD}$ group did have a significantly lower BMI $(p=0.03)$ and fat mass $(p<0.01)$ compared to the NC group (Table 1).

3.2. The Effect of Alzheimer's Disease on sRAGE Isoforms. There were no significant differences in total serum sRAGE between the AD and NC groups; however, individuals with $\mathrm{AD}$ had a significantly reduced cRAGE : esRAGE ratio $(p=$ 0.01 ) compared to those with NC (Figures 1(a) and 1(d)). Although the cRAGE and esRAGE isoforms alone were not significantly different between groups $(p=0.07$ and 0.79 , resp.), trending suggests that the $14.4 \%$ attenuation in the ratio is likely due to a preferential loss in cRAGE rather than esRAGE (Figures 1(b) and 1(c)).

3.3. The Effect of AD, Age, and APOE4 Genotype on sRAGE Isoforms. Given the altered sRAGE profile with AD presented in Figure 1, we aimed to identify any effects that age or genotype may have on serum sRAGE isoforms. First, stratification by decade of age $(60-69,70-79,80-89 y)$ revealed a main effect of AD on cRAGE $(p=0.03)$ but no significant differences between groups for total sRAGE, esRAGE, or the cRAGE: esRAGE ratio. We next stratified by APOE4 genotype, the primary risk gene in $\mathrm{AD}$ [34], and found significant main effects of AD on total sRAGE, cRAGE, and the cRAGE: esRAGE ratio $(p=0.05,0.02$, and 0.01$)$. There were no main effects of age or genotype nor any interactions between the above factors and AD status on any of the sRAGE variables. These findings suggest that age and APOE4 genotype may be important factors in the diminished cRAGE with AD.

3.4. The Effect of $A D$ and Metabolic Dysfunction on sRAGE Isoforms. To better characterize the effects of metabolic factors on sRAGE pathophysiology in $\mathrm{AD}$, all participants were stratified by the following factors: T2DM diagnosis, FGS as determined by ADA criteria, obesity by BMI according to the WHO, median insulin, median amylin, median HOMA-IR, and median fat mass. The above factors were selected for investigation due to their independent importance in disease pathophysiology and sRAGE biology. For example, we have previously found that glucose tolerance and obesity have a strong influence on sRAGE [29] and that adiposity (fat mass) is associated with attenuated sRAGE pools [35]. Further, this project aimed to characterize sRAGE profiles by cognitive status and commonly used markers of metabolic health to serve as a foundation for future work.

Total serum sRAGE and esRAGE were not significantly different between individuals with or without T2DM comorbid with AD. However, there was a significant main effect of $\mathrm{AD}$ on serum cRAGE and the cRAGE: esRAGE ratio $(p=0.03,0.03)$, showing decreased levels of these sRAGE isoforms with AD (data not shown). Similarly, when stratified by FGS, there was a main effect of $A D$,
TABLE 1: Subject characteristics.

\begin{tabular}{lcc}
\hline Variable (units) & $\mathrm{NC}(n=68)$ & $\mathrm{AD}(n=67)$ \\
\hline Sex (number, \% males) & $26,38 \%$ & $36,54 \%$ \\
Age (y) & $73 \pm 1$ & $75 \pm 1$ \\
BMI (kg/m $\left.{ }^{2}\right)$ & $29.5 \pm 0.5$ & $27.3 \pm 0.8^{*}$ \\
Lean body mass (kg) & $46 \pm 1$ & $46 \pm 1$ \\
Fat mass (kg) & $32 \pm 1$ & $27 \pm 1^{*}$ \\
Type 2 diabetes (number, \%) & $22,32 \%$ & $19,28 \%$ \\
HOMA-IR (AU) & $2.4 \pm 0.3$ & $2.2 \pm 0.2$ \\
Fasting glucose (mg/dL) & $104 \pm 3$ & $101 \pm 2$ \\
Fasting glucose (mmol/L) & $5.8 \pm 0.2$ & $5.6 \pm 0.1$ \\
Fasting insulin (mU/L) & $9.1 \pm 1.0$ & $8.8 \pm 0.8$ \\
Fasting amylin (pM) & $11.2 \pm 1.7$ & $17.9 \pm 5.3$ \\
S100B (pg/mL) & $13.0 \pm 1.7$ & $14.4 \pm 2.8$ \\
\hline
\end{tabular}

Data are presented as mean \pm SEM. BMI: body mass index; HOMA-IR: homeostatic model assessment of insulin resistance. ${ }^{*} p<0.05$.

which highlighted lower total sRAGE and cRAGE compared to NC counterparts ( $p=0.04,0.02$; data not shown).

Obesity stratification by BMI revealed main effects of $\mathrm{AD}$ on total sRAGE, cRAGE, and cRAGE: esRAGE ratio $(p=0.01,<0.01$, and 0.02$)$, main effects of obesity status on total sRAGE and cRAGE $(p=0.02,0.01)$, and interaction effects on total sRAGE, esRAGE, and cRAGE $(p=0.02,0.02$, and 0.02). Post hoc analysis showed that within the NC group, those who are overweight have significantly lower total sRAGE, cRAGE, and esRAGE than their lean counterparts $(p=0.01,0.02$, and 0.02 ; Figure 2). Similarly, within the $\mathrm{AD}$ group, obese individuals have significantly reduced cRAGE compared to lean individuals $(p=0.03$; Figure 2(b)). Those with comorbid obesity and AD have significantly lower total sRAGE and cRAGE compared to obese NC individuals ( $p=0.01,<0.01$; Figure 2$)$. When stratified by median fat mass $(28.95 \mathrm{~kg})$, the AD group had lower serum cRAGE and cRAGE: esRAGE $(p=0.04,0.02$; Figure 3). Interaction effects between $\mathrm{AD}$ status and fat mass highlighted significant attenuation of total sRAGE, cRAGE, and the cRAGE:esRAGE ratio with AD compared to NC in the high fat mass group $(p=0.01,<0.01$, and $<0.01$; Figure 3). As shown in Figure 3, individuals in the AD group with high fat mass had $4.1 \%$ less cRAGE and a $21.2 \%$ reduction in the cRAGE : esRAGE ratio compared to their counterparts with low fat mass $(p=0.3,0.02)$.

HOMA-IR was calculated as an indicator of insulin resistance, and participants were stratified by the calculated median (1.66 AU). This data showed a significant difference between the AD and NC groups in serum cRAGE and the cRAGE : esRAGE ratio ( $p=0.04,0.02$; Figure 4$)$. Additionally, within the high HOMA-IR group, individuals with concurrent $\mathrm{AD}$ had a $25.7 \%$ lower cRAGE: esRAGE ratio $(p<0.01$; Figure 4). Fasting HOMA-IR was also negatively correlated to log-transformed serum cRAGE $(r=-0.19$, $p=0.03$ ).

Insulin and amylin were found to have a similar influence on serum sRAGE profiles in AD, but amylin was of special interest due to its ability to colocalize with $\mathrm{A} \beta$ plaques in 


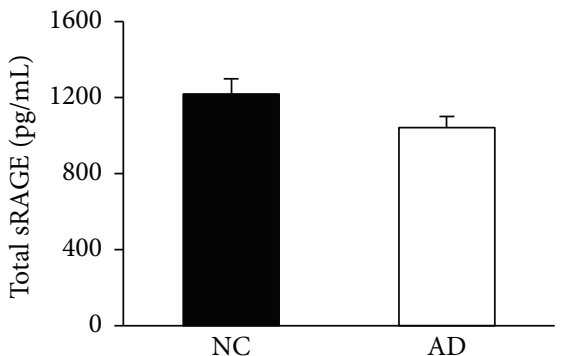

(a)

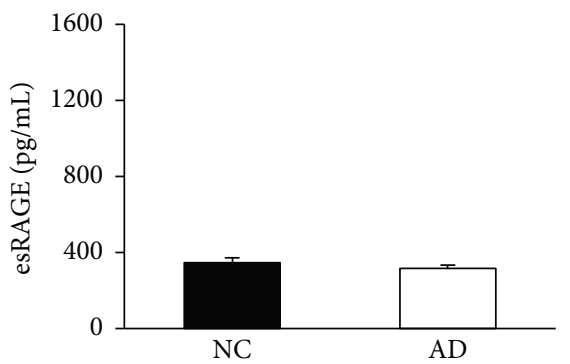

(c)

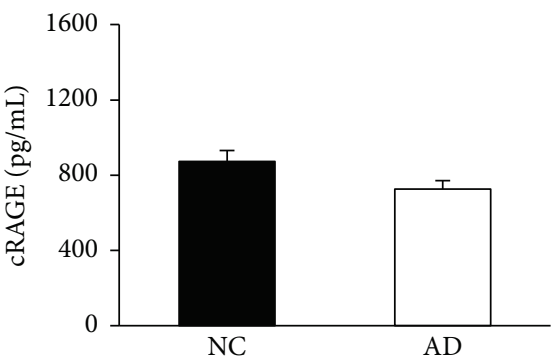

(b)

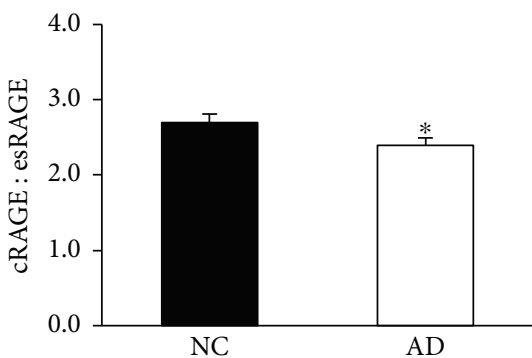

(d)

FIGURE 1: Attenuated cRAGE: esRAGE ratio with Alzheimer's disease compared to individuals with normal cognition. (a) Total sRAGE and (c) esRAGE were measured using commercially available ELISAs. (b) cRAGE was calculated by subtracting esRAGE from total sRAGE and the (d) cRAGE : esRAGE ratio was calculated by division. Data are presented as mean \pm SEM. Sample sizes for the stratifications are as follows: $\mathrm{NC}(n=68)$ and $\mathrm{AD}(n=67) .{ }^{*} p<0.05$ versus NC.

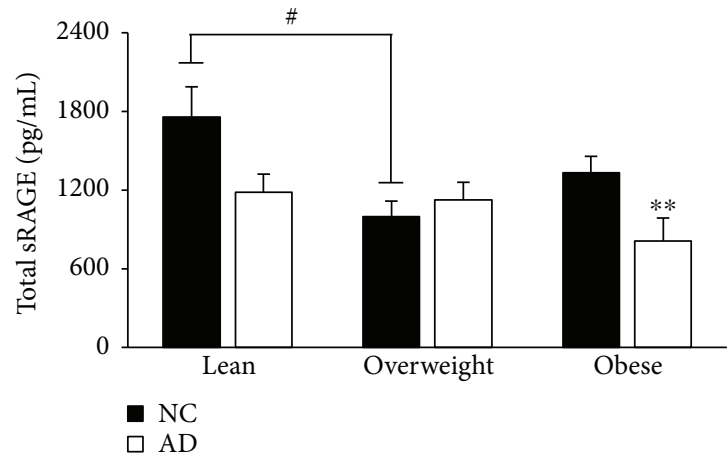

(a)

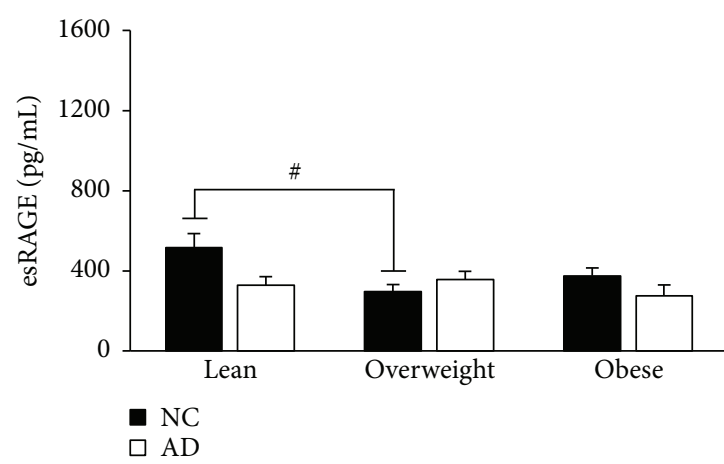

(c)

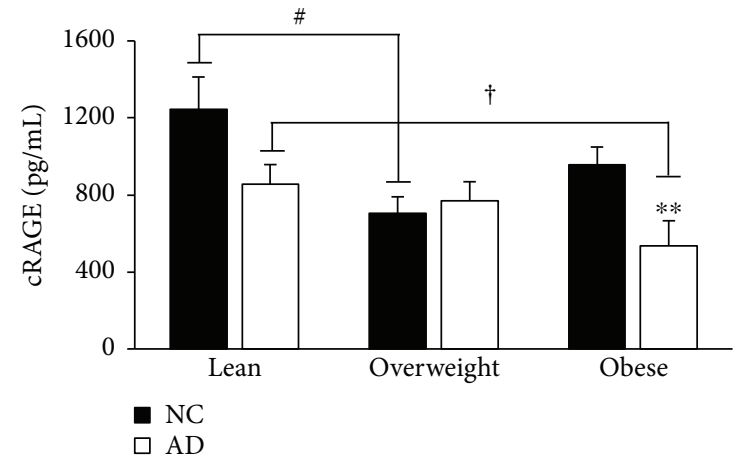

(b)

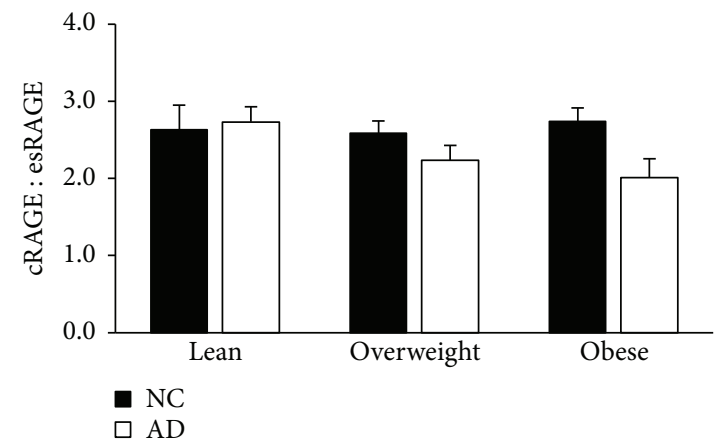

(d)

FIGURE 2: Diminished sRAGE pools are characteristic of individuals with concurrent AD and obesity. Participants were grouped by obesity status (lean: $<25 \mathrm{~kg} / \mathrm{m}^{2}$, overweight: $25-29 \mathrm{~kg} / \mathrm{m}^{2}$, obese: $\geq 30 \mathrm{~kg} / \mathrm{m}^{2}$ ), and sRAGE isoforms were measured in $\mathrm{pg} / \mathrm{mL}$ as mentioned previously. Data are presented as mean \pm SEM. Sample sizes for the stratifications are as follows: lean, NC $(n=7)$, lean, AD $(n=19)$, overweight, NC $(n=27)$, overweight, $\mathrm{AD}(n=20)$, obese, $\mathrm{NC}(n=24)$, and obese, $\mathrm{AD}(n=12) .{ }^{\#} p$ value $<0.05$ across metabolic factor within NC group; ${ }^{\dagger} p$ value $<0.05$ across metabolic factor within $\mathrm{AD}$ group; ${ }^{* *} p$ value $<0.01$ across groups within metabolic factor. 

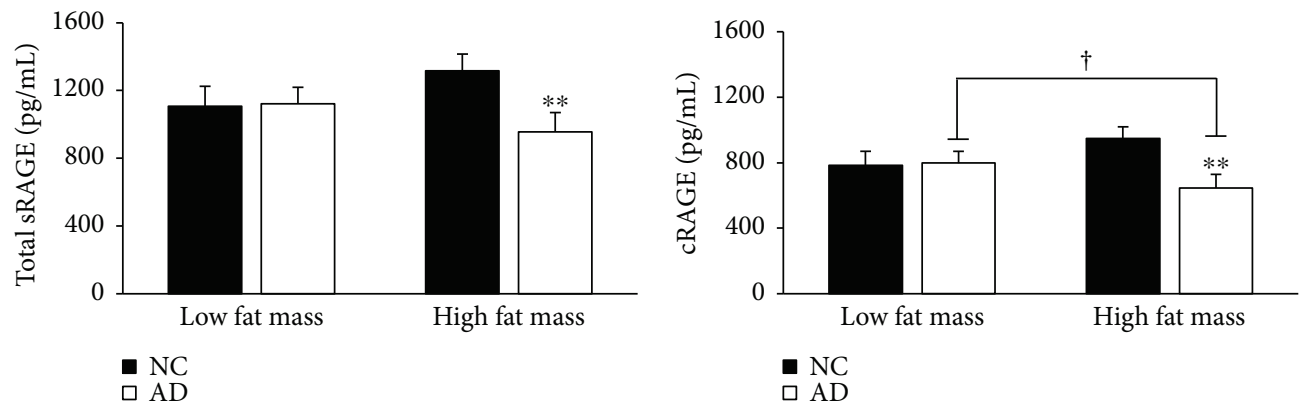

(a)

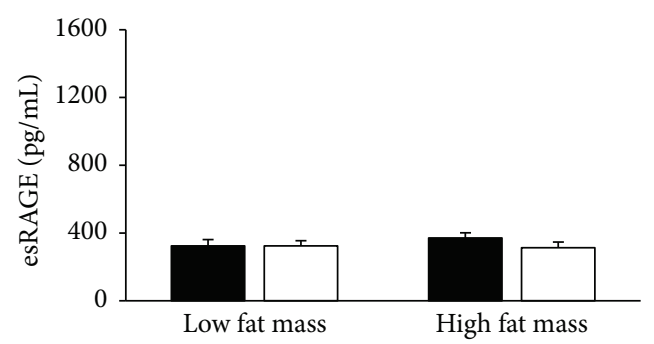

- NC

$\square \mathrm{AD}$

(b)

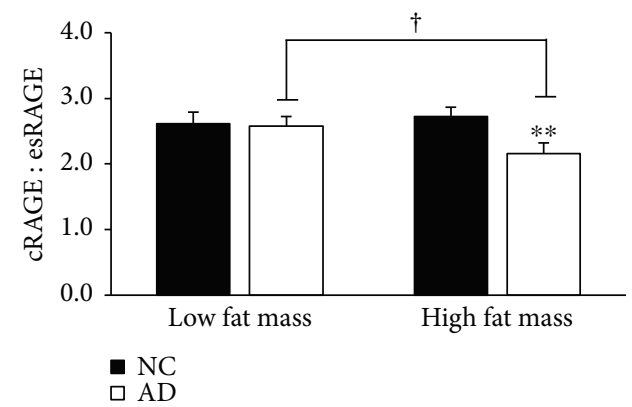

(c)

(d)

FIGURE 3: AD with high fat mass revealed marked reductions in total sRAGE and cRAGE and cRAGE: esRAGE compared to NC with high fat mass. Participants were stratified by median fat mass $(29 \mathrm{~kg})$ measured via DEXA. Data are presented as mean \pm SEM. Sample sizes for the stratifications are as follows: low fat mass, $\mathrm{NC}(n=26)$, low fat mass, $\mathrm{AD}(n=37)$, high fat mass, NC $(n=37)$, and high fat mass, $\mathrm{AD}(n=28) .{ }^{\dagger} p$ value $<0.05$ across metabolic factor within $\mathrm{AD}$ group; ${ }^{* *} p$ value $<0.01$ across groups within metabolic factor.

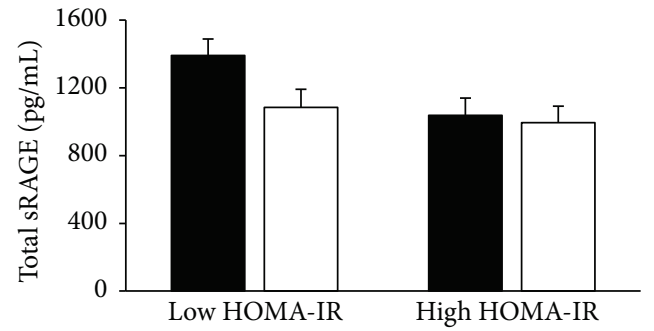

- NC

$\square \mathrm{AD}$

(a)

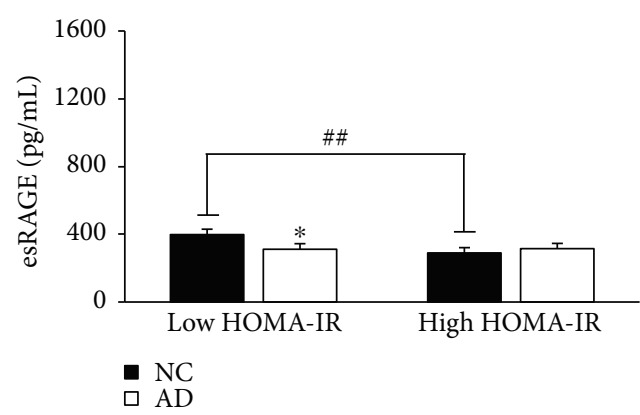

(c)

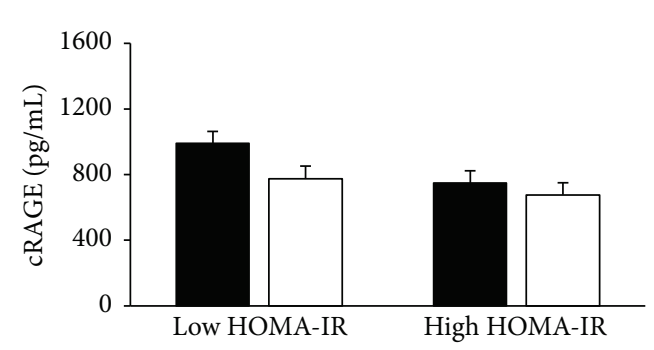

- NC

$\square \mathrm{AD}$

(b)

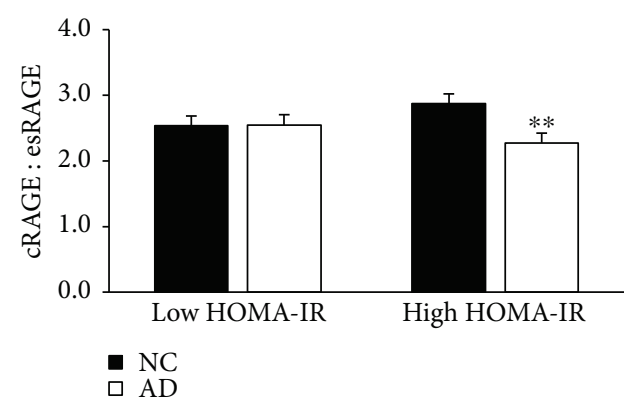

(d)

FIgURE 4: Decreased esRAGE and cRAGE : esRAGE ratio with high HOMA-IR or AD. HOMA-IR was calculated from fasting measures of glucose and insulin, and participants were stratified by the median HOMA-IR (1.7 AU). Data are presented as mean \pm SEM. Sample sizes for the stratifications are as follows: low HOMA-IR, NC $(n=35)$, low HOMA-IR, AD $(n=30)$, high HOMA-IR, NC ( $n=33)$, and high HOMA-IR, AD $(n=35) .{ }^{\# \#} p$ value $<0.01$ across metabolic factor within NC group; ${ }^{*} p$ value $<0.05$ across groups within metabolic factor; ${ }^{* *} p$ value $<0.01$ across groups within metabolic factor. 


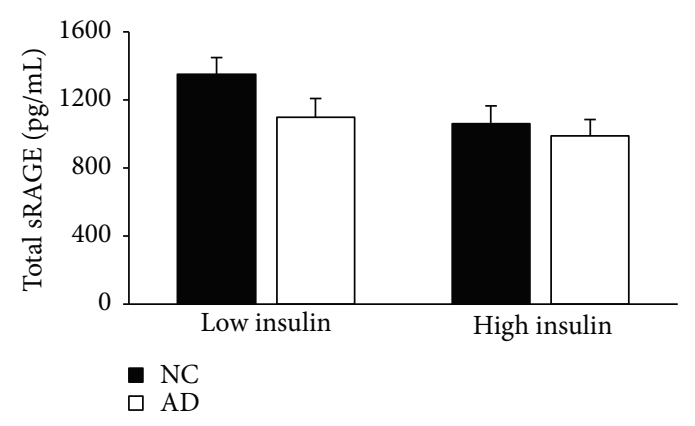

(a)

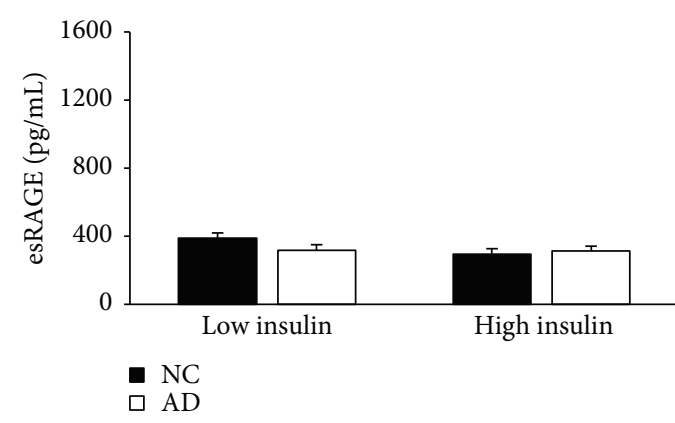

(c)

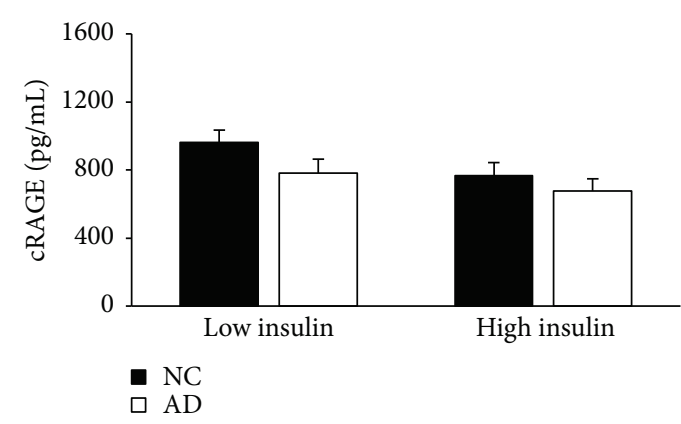

(b)

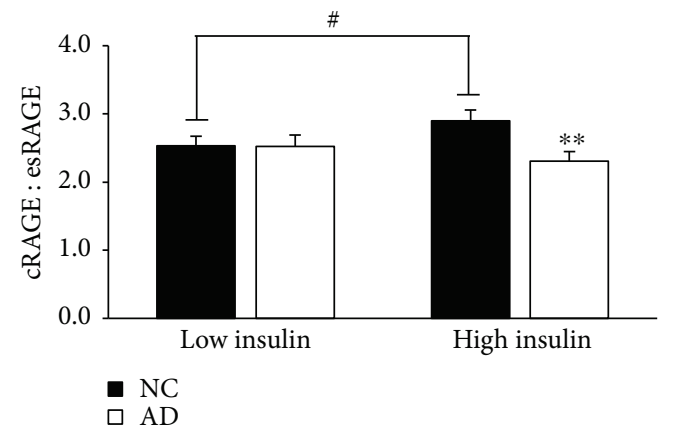

(d)

FIGURE 5: Individuals with high insulin concurrent with AD display attenuated cRAGE: esRAGE. Serum insulin was measured in mU/L following an overnight fast, and participants were stratified by the calculated median $(6.83 \mathrm{mU} / \mathrm{L})$. Data are presented as mean \pm SEM. Sample sizes for the stratifications are as follows: low insulin, NC $(n=37)$, low insulin, AD $(n=31)$, high insulin, NC ( $n=28)$, and high insulin, $\mathrm{AD}(n=37) .{ }^{\#} p$ value $<0.05$ across metabolic factor within $\mathrm{NC}$ group; ${ }^{* *} p$ value $<0.01$ across groups within metabolic factor.

$\mathrm{AD}$ [36]. Outlined in Figures 5 and 6, when stratified by median insulin $(6.83 \mathrm{mU} / \mathrm{L})$ and median amylin $(6.75 \mathrm{pM})$, individuals with $\mathrm{AD}$ presented with lower $\mathrm{cRAGE}(p=0.04$, $0.03)$ and cRAGE: esRAGE $(p=0.01,0.01)$. In addition, there was an interaction between $\mathrm{AD}$ and both insulin group and amylin group ( $p=0.02,<0.01$; Figures 5 and 6$)$. Within both the high insulin and high amylin groups, those with concurrent $\mathrm{AD}$ displayed aberrant serum cRAGE: esRAGE ratios compared to NC counterparts, with a $25.6 \%$ and $30.1 \%$ attenuation, respectively $(p<0.01,<0.01)$. Related, fasting amylin was negatively correlated to log-transformed values of both the esRAGE $(r=-0.31, p<0.01)$ and the cRAGE isoforms $(r=-0.29, p<0.01)$. Fasting insulin was also negatively correlated to log-transformed serum cRAGE $(r=-0.19, p=0.03)$.

To understand any influence that the well-established factors of age and sex may have on AD-related outcomes, we ran ANCOVAs for these factors. All of the significant interactions we found between the experimental metabolic factors of interest and $\mathrm{AD}$ status on sRAGE profiles were maintained with covariance for age and sex (data not shown).

3.5. The Effect of AD on the sRAGE Ligand S100B. There were no significant differences in serum S100B between the $\mathrm{AD}$ and $\mathrm{NC}$ groups (Table 1). Ratios between serum S100B and serum total sRAGE, esRAGE, and cRAGE were generated to investigate RAGE ligand and sRAGE dynamics. Analyses showed no significant differences across AD diagnosis (data not shown). Additionally, there were no significant correlations among S100B, total sRAGE, or any of the sRAGE isoforms. We further investigated serum S100B against determinants of metabolic health (fat mass, BMI, insulin, glucose, and HOMA-IR) and found no significant relationships.

\section{Discussion}

To our knowledge, this is the first report to characterize total sRAGE and all sRAGE isoforms in individuals with and without Alzheimer's disease and comorbid T2DM. Our data show that individuals with metabolic derangement (elevated insulin, amylin, HOMA-IR, BMI, or fat mass) concurrent with $\mathrm{AD}$ present with aberrant sRAGE profiles. These findings are in line with other published work that shows decreased plasma sRAGE in individuals with cognitive impairment, obesity, and IR [26, 30,37] and further supports the protective role of sRAGE in inflammatory diseases.

Novel findings of importance were the alterations in the sRAGE profile when individuals were stratified by AD diagnosis and median amylin. Fasting amylin was also negatively correlated to both esRAGE and cRAGE. Amylin readily crosses the blood-brain barrier and is known to play an important role in mediating brain function [38]. Further, amylin and $\mathrm{A} \beta$ share many chemical features, as both molecules bind to the same amylin-3 receptor in the brain [39]. Under pathological conditions, amylin can form insoluble amylin amyloid plaques, which have been found to colocalize with $\mathrm{A} \beta$ plaques in $\mathrm{AD}$ [36]. Abnormal amylin is central to $\mathrm{T} 2 \mathrm{DM}$ progression as it is seen in both early stages (elevated 


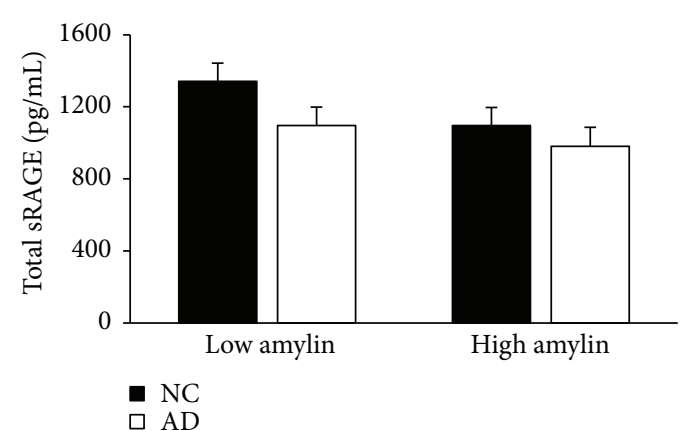

(a)

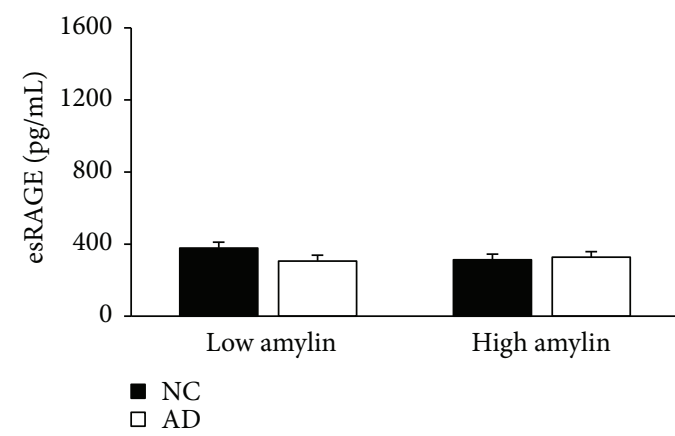

(c)

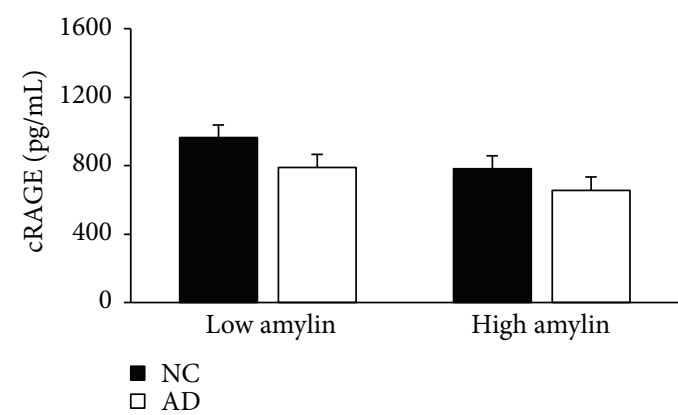

(b)

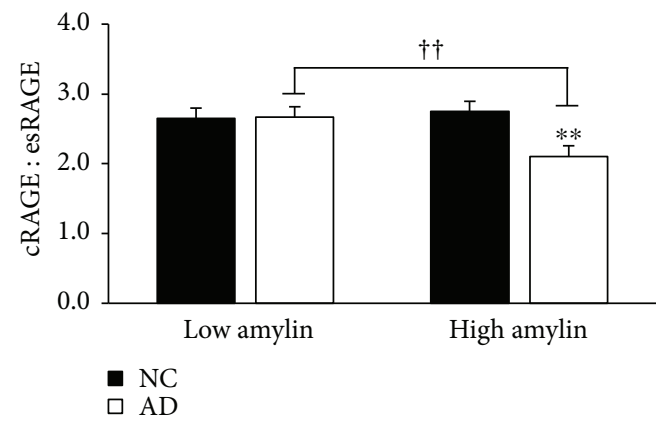

(d)

FIGURe 6: Aberrant cRAGE: esRAGE in individuals with high amylin and comorbid AD. Serum amylin was measured in pM following an overnight fast, and participants were stratified by the calculated median $(6.8 \mathrm{pM})$. Data are presented as mean \pm SEM. Sample sizes for the stratifications are as follows: low HOMA-IR, NC $(n=34)$, low HOMA-IR, AD $(n=34)$, high HOMA-IR, NC $(n=34)$, and high HOMA-IR, $\mathrm{AD}(n=32) .{ }^{\dagger \dagger} p$ value $<0.01$ across metabolic factor within $\mathrm{AD}$ group; ${ }^{* *} p$ value $<0.01$ across groups within metabolic factor.

amylin) and late stages (suppressed amylin) of the disease [40]. This, combined with the known relationship between RAGE and neurodegenerative disorders, highlights the link between abnormal amylin, depressed sRAGE, and Alzheimer's disease seen in our results.

We also showed that when our analyses were covaried for both age and sex, all interaction effects between metabolic dysfunction and AD status remained significant. Similarly, when individuals were stratified by decade of age or APOE4 genotype, there were neither any main effects of these variables nor an interaction with AD status. Although APOE4 genotype and increasing age are well-established risk factors for late-onset $\mathrm{AD}$, previous studies have found that total plasma sRAGE levels are able to predict cognitive decline seen in late-onset $\mathrm{AD}$ [30]. Together with our data, these findings suggest that widespread metabolic dysfunction, rather than more traditional risk factors, may be more important drivers of $\mathrm{AD}$ in some individuals.

However, when we examined sRAGE in individuals with $\mathrm{AD}, \mathrm{T} 2 \mathrm{DM}$ status did not impact total sRAGE or any specific isoforms. This differs from previous work out of our lab and others that suggest diminished sRAGE pools in individuals with T2DM compared to healthy controls $[29,41]$. There is also conflicting evidence demonstrating that increased total sRAGE is associated with diabetes [42]. One possible explanation for this difference may be that in the present, all study participants remained on their T2DM medication, whereas Basta et al. only included T2DM subjects without medication [41]. Another possible explanation is that many of these volunteers may be newly diagnosed with T2DM or have adequate glycemic control as the median HOMA-IR of our T2DM participants was 2.45, much lower than previous established cutoffs [43]. Lastly, it may be possible that $\mathrm{AD}$ pathology may present similarly to T2DM and thus may mask any unique effects of T2DM on sRAGE isoforms.

$\mathrm{S} 100 \mathrm{~B}$ is thought to mediate neuroinflammation and contribute to both $\mathrm{AD}$ pathogenesis and systemic inflammation seen in metabolic disease. Importantly, S100B is overexpressed in the brain of $\mathrm{AD}$ patients $[44,45]$ and elevated in serum of persons with obesity [46]. However, reports of S100B in cerebral spinal fluid (CSF) and serum of $\mathrm{AD}$ patients are conflicting. Peskind et al. reported elevated CSF $\mathrm{S} 100 \mathrm{~B}$ in individuals with mild to moderate $\mathrm{AD}$ compared to controls [47], while Nooijen et al. found no difference in CSF S100B with dementia [48]. Chaves et al. reported lower serum S100B in AD compared to controls [49], which differs with our cross-sectional analysis, as we did not see any differences in serum S100B across AD status. A possible explanation of our findings is that elevated RAGE in the $\mathrm{AD}$ brain sequesters and traps S100B proteins in brain tissue, preventing it from appearing in the serum despite the compromised blood-brain barrier in AD. Similar RAGE trapping mechanisms have been previously reported in adipose tissue for advanced glycated end products [50]. Further, adipose tissue serves as a site of $\mathrm{S} 100 \mathrm{~B}$ protein concentration comparable to neuronal tissue [51,52]. S100B secretion from adipocytes is related to lipolysis [53], inhibited by insulin 
[54], and thus is subject to dysregulation by diabetes and other metabolic diseases. This may further explain our findings as we balanced the number of T2DM participants across cognitive groups. These data further illuminate the need to consider metabolic covariates when assessing neurocognitive disorders. Postmortem analysis of brain sections combined with CSF and serum analyses may help to clarify the role and origin of $\mathrm{S} 100 \mathrm{~B}$ in the pathogenesis of $\mathrm{AD}$.

In conclusion, the following metabolic factors have been shown to influence total sRAGE and individual sRAGE isoforms in Alzheimer's disease: elevated insulin, amylin, insulin resistance as described by HOMA-IR, obesity by BMI, and high fat mass. It is possible that the attenuated plasma sRAGE seen in individuals with metabolic dysfunction may be contributing to $\mathrm{AD}$ due to a reduced capacity to scavenge RAGE ligands and attenuate RAGE signaling. Activation of RAGE produces oxidative stress and inflammation in the brain, neuronal degeneration, and $\mathrm{A} \beta$ accumulation, all inducers of cognitive impairment and $\mathrm{AD}[10,20]$. These novel characterizations of sRAGE in individuals with concurrent metabolic derangement and $\mathrm{AD}$ contribute evidence to the link between impaired metabolism and cognitive decline.

\section{Conflicts of Interest}

The authors declare that there is no conflict of interest regarding the publication of this article.

\section{Acknowledgments}

This work was supported by the National Institutes of Health [R01 DK109948 (Jacob M. Haus), K99 AG050490 (Jill K. Morris), and P30AG035982 Jill K. Morris)].

\section{References}

[1] J. A. Luchsinger and D. R. Gustafson, "Adiposity, type 2 diabetes, and Alzheimer's disease," Journal of Alzheimer's Disease, vol. 16, no. 4, pp. 693-704, 2009.

[2] J. A. Luchsinger, M.-X. Tang, S. Shea, and R. Mayeux, "Hyperinsulinemia and risk of Alzheimer disease," Neurology, vol. 63, no. 7, pp. 1187-1192, 2004.

[3] S. Hoyer, "Glucose metabolism and insulin receptor signal transduction in Alzheimer disease," European Journal of Pharmacology, vol. 490, no. 1-3, pp. 115-125, 2004.

[4] A. M. Rawlings, A. R. Sharrett, T. H. Mosley, S. H. Ballew, J. A. Deal, and E. Selvin, "Glucose peaks and the risk of dementia and 20-year cognitive decline," Diabetes Care, vol. 40, no. 7 , pp. 879-886, 2017.

[5] S. M. de la Monte and M. Tong, "Brain metabolic dysfunction at the core of Alzheimer's disease," Biochemical Pharmacology, vol. 88, no. 4, pp. 548-559, 2014.

[6] Z. Chen and C. Zhong, "Decoding Alzheimer's disease from perturbed cerebral glucose metabolism: implications for diagnostic and therapeutic strategies," Progress in Neurobiology, vol. 108, pp. 21-43, 2013.

[7] S. Craft, "The role of metabolic disorders in Alzheimer disease and vascular dementia: two roads converged?" Archives of Neurology, vol. 66, no. 3, pp. 300-305, 2009.
[8] R. I. Fink, O. G. Kolterman, J. Griffin, and J. M. Olefsky, "Mechanisms of insulin resistance in aging," Journal of Clinical Investigation, vol. 71, no. 6, pp. 1523-1535, 1983.

[9] W. A. Rocca, A. Hofman, C. Brayne et al., "Frequency and distribution of Alzheimer's disease in Europe: a collaborative study of 1980-1990 prevalence findings," Annals of Neurology, vol. 30, no. 3, pp. 381-390, 1991.

[10] Z. Cai, N. Liu, C. Wang et al., "Role of RAGE in Alzheimer's disease," Cellular and Molecular Neurobiology, vol. 36, no. 4, pp. 483-495, 2016.

[11] L. Sessa, E. Gatti, F. Zeni et al., "The receptor for advanced glycation end-products (RAGE) is only present in mammals, and belongs to a family of cell adhesion molecules (CAMs)," PLoS One, vol. 9, no. 1, article e86903, 2014.

[12] M. Neeper, A. M. Schmidt, J. Brett et al., "Cloning and expression of a cell surface receptor for advanced glycosylation end products of proteins," Journal of Biological Chemistry, vol. 267, no. 21, pp. 14998-15004, 1992.

[13] L. J. Sparvero, D. Asafu-Adjei, R. Kang et al., "RAGE (receptor for advanced glycation endproducts), RAGE ligands, and their role in cancer and inflammation," Journal of Translational Medicine, vol. 7, no. 1, p. 17, 2009.

[14] N. Taniguchi, M. Takahashi, Y. Kizuka et al., "Glycation vs. glycosylation: a tale of two different chemistries and biology in Alzheimer's disease," Glycoconjugate Journal, vol. 33, no. 4, pp. 487-497, 2016.

[15] M. C. Miller, R. Tavares, C. E. Johanson et al., "Hippocampal RAGE immunoreactivity in early and advanced Alzheimer's disease," Brain Research, vol. 1230, pp. 273-280, 2008.

[16] R. E. Mrak and W.S. Griffinbc, "The role of activated astrocytes and of the neurotrophic cytokine S100B in the pathogenesis of Alzheimer's disease," Neurobiology of Aging, vol. 22, no. 6, pp. 915-922, 2001.

[17] C. Cirillo, E. Capoccia, T. Iuvone et al., "S100B inhibitor pentamidine attenuates reactive gliosis and reduces neuronal loss in a mouse model of Alzheimer's disease," BioMed Research International, vol. 2015, Article ID 508342, 11 pages, 2015.

[18] A. M. Schmidt, S. D. Yan, S. F. Yan, and D. M. Stern, "The multiligand receptor RAGE as a progression factor amplifying immune and inflammatory responses," Journal of Clinical Investigation, vol. 108, no. 7, pp. 949-955, 2001.

[19] V. P. Singh, A. Bali, N. Singh, and A. S. Jaggi, "Advanced glycation end products and diabetic complications," The Korean Journal of Physiology \& Pharmacology, vol. 18, no. 1, pp. 1-14, 2014.

[20] G. Perry, A. D. Cash, and M. A. Smith, "Alzheimer disease and oxidative stress," Journal of Biomedicine and Biotechnology, vol. 2, no. 3, pp. 120-123, 2002.

[21] E. Kosenko, G. Aliev, L. Tikhonova, Y. Li, A. Poghosyan, and Y. Kaminsky, "Antioxidant status and energy state of erythrocytes in Alzheimer dementia: probing for markers," CNS \& Neurological Disorders - Drug Targets, vol. 11, no. 7, pp. 926-932, 2012.

[22] J. Pozueta, R. Lefort, and M. L. Shelanski, "Synaptic changes in Alzheimer's disease and its models," Neuroscience, vol. 251, pp. 51-65, 2013.

[23] A. Raucci, S. Cugusi, A. Antonelli et al., "A soluble form of the receptor for advanced glycation endproducts (RAGE) is produced by proteolytic cleavage of the membrane-bound form by the sheddase a disintegrin and metalloprotease 10 
(ADAM10)," The FASEB Journal, vol. 22, no. 10, pp. 37163727, 2008.

[24] H. Yonekura, Y. Yamamoto, S. Sakurai et al., "Novel splice variants of the receptor for advanced glycation end-products expressed in human vascular endothelial cells and pericytes, and their putative roles in diabetes-induced vascular injury," Biochemical Journal, vol. 370, no. 3, pp. 1097-1109, 2003.

[25] S. Zeng, N. Feirt, M. Goldstein et al., "Blockade of receptor for advanced glycation end product (RAGE) attenuates ischemia and reperfusion injury to the liver in mice," Hepatology, vol. 39, no. 2, pp. 422-432, 2004.

[26] E. Dozio, S. Briganti, A. Delnevo et al., "Relationship between soluble receptor for advanced glycation end products (sRAGE), body composition and fat distribution in healthy women," European Journal of Nutrition, vol. 56, no. 8, pp. 2557-2564, 2017.

[27] T. M. Motawi, M. A. Abou-Seif, A. M. Bader, and M. O. Mahmoud, "Effect of glycemic control on soluble RAGE and oxidative stress in type 2 diabetic patients," BMC Endocrine Disorders, vol. 13, no. 1, 2013.

[28] E. Emanuele, A. D’Angelo, C. Tomaino et al., "Circulating levels of soluble receptor for advanced glycation end products in Alzheimer disease and vascular dementia," Archives of Neurology, vol. 62, no. 11, pp. 1734-1736, 2005.

[29] E. R. Miranda, V. S. Somal, J. T. Mey et al., "Circulating soluble RAGE isoforms are attenuated in obese, impaired-glucosetolerant individuals and are associated with the development of type 2 diabetes," American Journal of PhysiologyEndocrinology and Metabolism, vol. 313, no. 6, pp. E631E640, 2017.

[30] R. Ghidoni, L. Benussi, M. Glionna et al., "Decreased plasma levels of soluble receptor for advanced glycation end products in mild cognitive impairment," Journal of Neural Transmission, vol. 115, no. 7, pp. 1047-1050, 2008.

[31] X. Y. Xu, C. Q. Deng, J. Wang et al., "Plasma levels of soluble receptor for advanced glycation end products in Alzheimer's disease," International Journal of Neuroscience, vol. 127, no. 5, pp. 454-458, 2017.

[32] B. Singh and A. Saxena, "Surrogate markers of insulin resistance: a review," World Journal of Diabetes, vol. 1, no. 2, pp. 36-47, 2010.

[33] S. C. Tang, S. J. Yeh, L. K. Tsai et al., "Cleaved but not endogenous secretory RAGE is associated with outcome in acute ischemic stroke," Neurology, vol. 86, no. 3, pp. 270276, 2016.

[34] E. M. Reiman, K. Chen, X. Liu et al., "Fibrillar amyloid- $\beta$ burden in cognitively normal people at 3 levels of genetic risk for Alzheimer's disease," Proceedings of the National Academy of Sciences of the United States of America, vol. 106, no. 16, pp. 6820-6825, 2009.

[35] E. Miranda, K. Varady, and J. Haus, "Weight loss via alternate day fasting increases circulating endogenous secretory RAGE and is associated with markers of adipocyte health," The FASEB Journal, vol. 31, p. 3, 2017.

[36] K. Jackson, G. A. Barisone, E. Diaz, L. W. Jin, C. DeCarli, and F. Despa, "Amylin deposition in the brain: a second amyloid in Alzheimer disease?," Annals of Neurology, vol. 74, no. 4, pp. 517-526, 2013.

[37] I. Hagen, D. M. Schulte, N. Müller et al., "Soluble receptor for advanced glycation end products as a potential biomarker to predict weight loss and improvement of insulin sensitivity by a very low calorie diet of obese human subjects," Cytokine, vol. 73, no. 2, pp. 265-269, 2015.

[38] W. Q. Qiu, H. Li, H. Zhu et al., "Plasma amylin and cognition in diabetes in the absence and the presence of insulin treatment," Journal of Diabetes \& Metabolism, vol. 5, no. 11, 2014.

[39] W. Fu, A. Ruangkittisakul, D. MacTavish, J. Y. Shi, K. Ballanyi, and J. H. Jhamandas, "Amyloid $\beta(\mathrm{A} \beta)$ peptide directly activates amylin-3 receptor subtype by triggering multiple intracellular signaling pathways," Journal of Biological Chemistry, vol. 287, no. 22, pp. 18820-18830, 2012.

[40] T. A. Lutz and U. Meyer, "Amylin at the interface between metabolic and neurodegenerative disorders," Frontiers in Neuroscience, vol. 9, no. 216, 2015.

[41] G. Basta, A. M. Sironi, G. Lazzerini et al., "Circulating soluble receptor for advanced glycation end products is inversely associated with glycemic control and S100A12 protein," The Journal of Clinical Endocrinology \& Metabolism, vol. 91, no. 11, pp. 4628-4634, 2006.

[42] K. C. B. Tan, S. W. M. Shiu, W. S. Chow, L. Leng, R. Bucala, and D. J. Betteridge, "Association between serum levels of soluble receptor for advanced glycation end products and circulating advanced glycation end products in type 2 diabetes," Diabetologia, vol. 49, no. 11, pp. 2756-2762, 2006.

[43] S. E. Stern, K. Williams, E. Ferrannini, R. A. DeFronzo, C. Bogardus, and M. P. Stern, "Identification of individuals with insulin resistance using routine clinical measurements," Diabetes, vol. 54, no. 2, pp. 333-339, 2005.

[44] E. Leclerc, E. Sturchler, and S. W. Vetter, "The S100B/RAGE axis in Alzheimer's disease," Cardiovascular Psychiatry and Neurology, vol. 2010, Article ID 539581, 11 pages, 2010.

[45] F. Michetti, V. Corvino, M. C. Geloso et al., "The S100B protein in biological fluids: more than a lifelong biomarker of brain distress," Journal of Neurochemistry, vol. 120, no. 5, pp. 644-659, 2012.

[46] J. Steiner, K. Schiltz, M. Walter et al., "S100B serum levels are closely correlated with body mass index: an important caveat in neuropsychiatric research," Psychoneuroendocrinology, vol. 35, no. 2, pp. 321-324, 2010.

[47] E. R. Peskind, W. S. Griffin, K. T. Akama, M. A. Raskind, and L. J. Van Eldik, "Cerebrospinal fluid S100B is elevated in the earlier stages of Alzheimer's disease," Neurochemistry International, vol. 39, no. 5-6, pp. 409-413, 2001.

[48] P. T. Nooijen, H. C. Schoonderwaldt, R. A. Wevers, O. R. Hommes, and K. J. Lamers, "Neuron-specific enolase, S-100 protein, myelin basic protein and lactate in CSF in dementia," Dementia and Geriatric Cognitive Disorders, vol. 8, no. 3, pp. 169-173, 1997.

[49] M. L. Chaves, A. L. Camozzato, E. D. Ferreira et al., "Serum levels of S100B and NSE proteins in Alzheimer's disease patients," Journal of Neuroinflammation, vol. 7, no. 1, p. 6, 2010.

[50] K. H. J. Gaens, G. H. Goossens, P. M. Niessen et al., " $\mathrm{N}^{\varepsilon}$ (Carboxymethyl)lysine-receptor for advanced glycation end product axis is a key modulator of obesity-induced dysregulation of adipokine expression and insulin resistance," Arteriosclerosis, Thrombosis, and Vascular Biology, vol. 34, no. 6, pp. 1199-1208, 2014.

[51] H. Hidaka, T. Endo, S. Kawamoto et al., "Purification and characterization of adipose tissue S-100b protein," Journal of Biological Chemistry, vol. 258, no. 4, pp. 2705-2709, 1983. 
[52] F. Michetti, E. Dell'Anna, G. Tiberio, and D. Cocchia, "Immunochemical and immunocytochemical study of S-100 protein in rat adipocytes," Brain Research, vol. 262, no. 2, pp. 352356, 1983.

[53] C. B. Netto, S. Conte, M. C. Leite et al., "Serum S100B protein is increased in fasting rats," Archives of Medical Research, vol. 37, no. 5, pp. 683-686, 2006.

[54] F. Suzuki and K. Kato, "Inhibition of adipose S-100 protein release by insulin," Biochimica et Biophysica Acta (BBA) Molecular Cell Research, vol. 845, no. 2, pp. 311-316, 1985. 


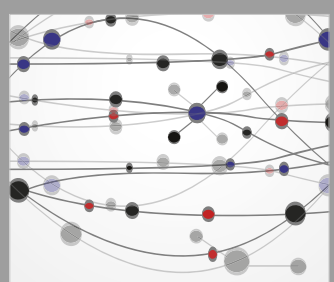

The Scientific World Journal
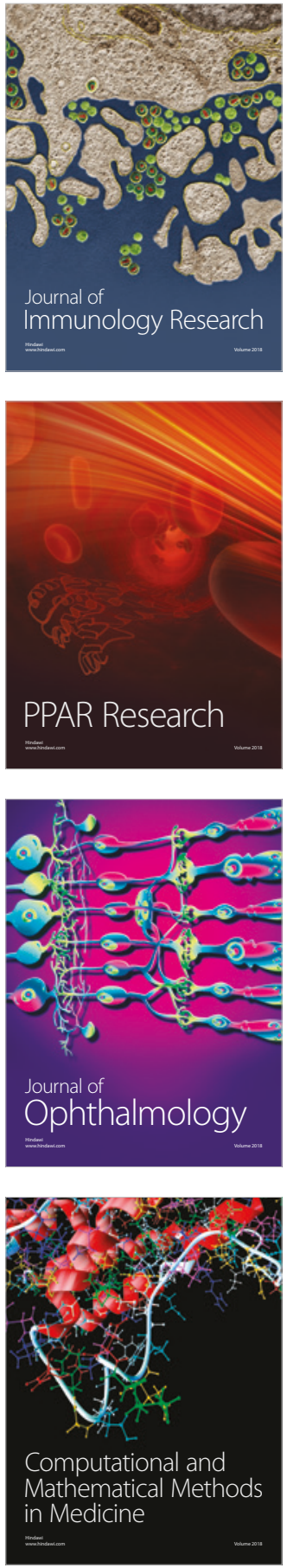

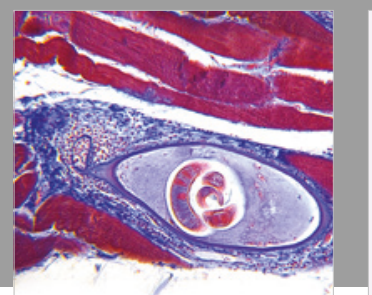

Gastroenterology Research and Practice

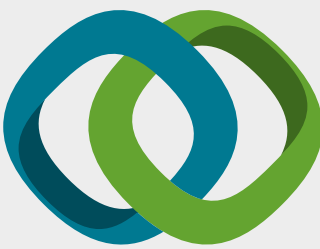

\section{Hindawi}

Submit your manuscripts at

www.hindawi.com
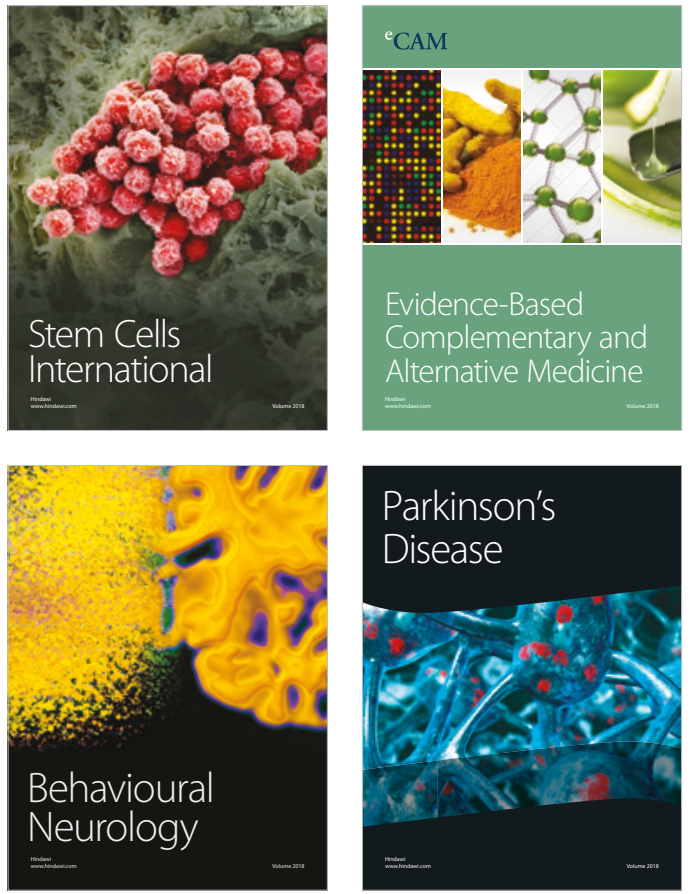

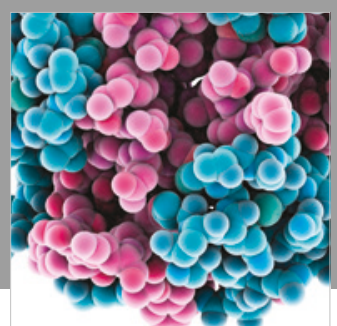

ournal of

Diabetes Research

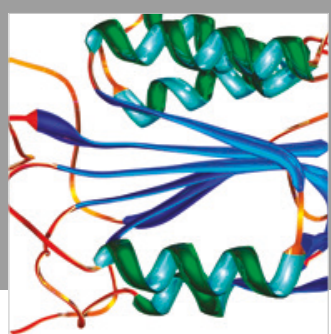

Disease Markers
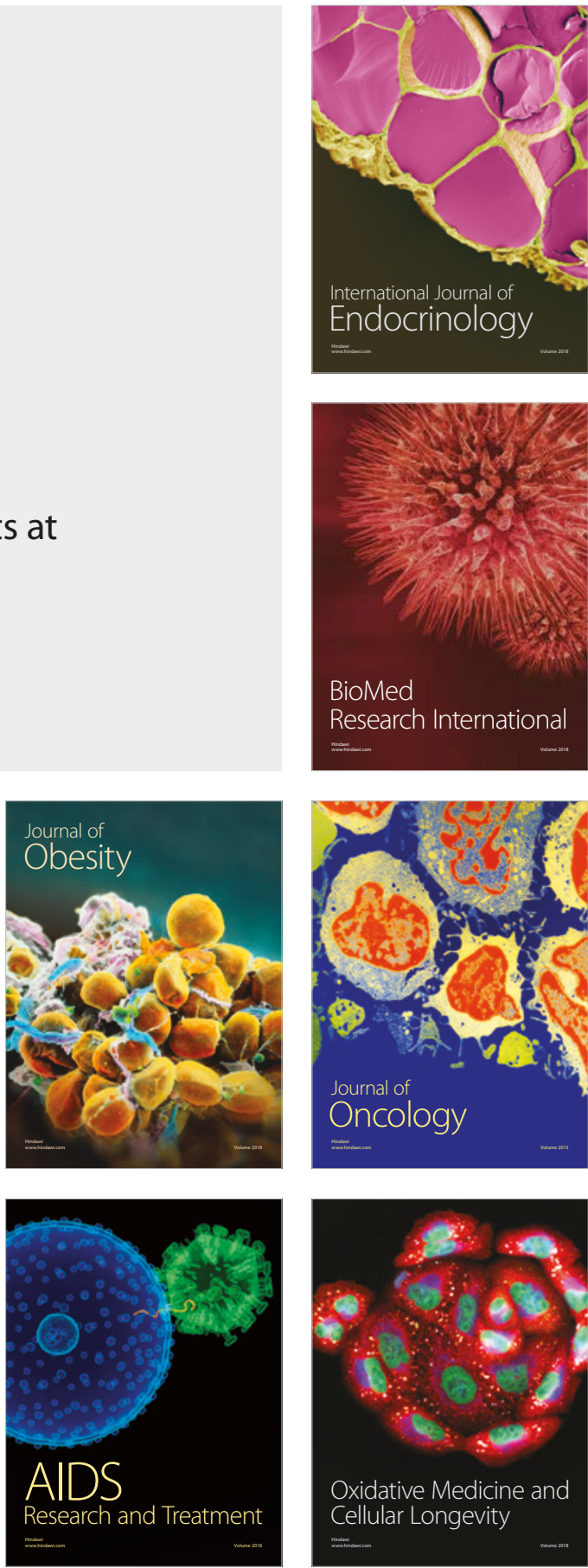